\title{
The value of measuring saccadic eye movement in the investigation of non-compressive myelopathy
}

\author{
M C PITT,* J M RAWLES $\dagger$
}

From Aberdeen Royal Infirmary, ${ }^{*}$ Foresterhill, and the Department of Medicine and Therapeutics, $\dagger$ University of Aberdeen, Aberdeen, Scotland

SUMMARY Saccadic eye movement recording was performed in 53 patients with non-compressive myelopathy. Twenty one patients $(40 \%)$ had subclinical abnormalities of saccadic movement, supporting a diagnosis of probable multiple sclerosis. When used in addition to the measurement of visual evoked potentials and brainstem auditory evoked responses, the detection of subclinical abnormalities increased from $40 \%$ to $57 \%$. The detection rate of abnormalities by saccadic eye movement recording was equal to that of visual evoked responses, but more than that of brainstem auditory evoked responses. Prolonged latency of gaze was the most common saccadic latency abnormality detected. The majority of saccadic velocity abnormalities could be explained by disease in the medial longitudinal bundle. An unusual finding was that abduction velocity was increased in six patients. It is concluded that the simple measurement of saccadic eye movement is a valuable addition to other ancillary investigations for the diagnosis of multiple sclerosis. It also allows analysis of oculomotor function, commonly disordered in multiple sclerosis, but rarely investigated.

In multiple sclerosis saccadic eye movement is frequently clinically abnormal, with a high incidence of internuclear ophthalmoplegia' resulting from involvement of the medial longitudinal bundle. ${ }^{2}$ Bipolar recording of the corneo-retinal potential is the simplest of several methods used for measuring saccadic eye movement, ${ }^{3}$ and offers no serious technical difficulties. Despite the potential for using such a technique for either confirming the presence of an abnormality suspected clinically or demonstrating subclinical involvement, few studies of patients with multiple sclerosis have been reported. ${ }^{46}$ Seldom has the measurement of saccadic eye movement been compared with other well established ancillary investigations in multiple sclerosis. ${ }^{78}$ The greatest potential value of this test is in patients suspected of having multiple sclerosis, in whom the presenting lesion is located outside the brainstem, as in noncompressive myelopathy. ${ }^{9} 10$

A prototype system for measuring saccadic latency

Correspondence to: Dr M C Pitt, Department of Neurophysiology, The National Hospital for Nervous Diseases, Queen Square, London WCIN 3BG, United Kingdom.

Received 19 September 1988.

Accepted 18 November 1988 and velocity has been developed, and is described in detail elsewhere, together with results from normal subjects." In this study we report results from 53 patients with non-compressive myelopathy and compare the diagnostic usefulness of saccadic eye movement recording (SEMR) with that of visual evoked potentials (VEP) and brainstem auditory evoked responses (BAER). We discuss the patterns of oculomotor abnormality found in a system frequently involved but not commonly investigated in multiple sclerosis.

\section{Patients and methods}

\section{Patients}

Fifty three patients with the diagnosis of non-compressive myelopathy were studied. By definition they had evidence of a myelopathy, such as spastic paraparesis or a BrownSequard lesion, no demonstrable cord compression on myelography and no definite clinical evidence of disease above the spinal cord. There were 29 females and 24 males, average age $52 \cdot 1$ years (SD 14:0).

\section{Ancillary investigations}

(a) Measurement of saccadic latency and velocity ${ }^{11}$

The corneo-retinal potentials generated from both eyes were detected with bipolar electrodes and simultaneously displayed on two twin-channel oscilloscopes, one of which was 

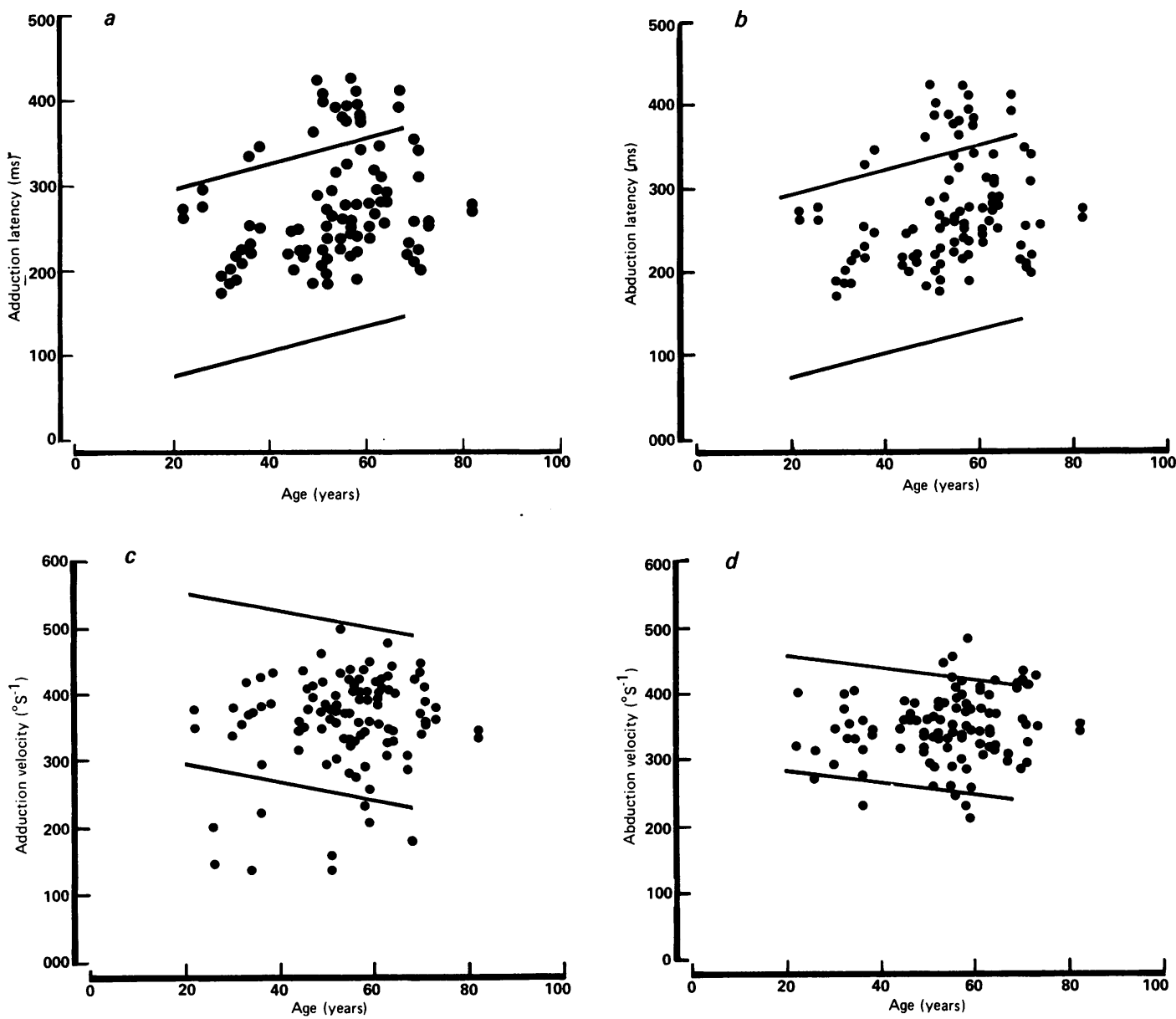

Fig (a) Adduction latency at various ages in 53 patients with non-compressive myelopathy, in relation to $99 \%$ confidence limits for healthy subjects, (b) as (a) for abduction latency, (c) as (a) for abduction velocity, (d) as (a) for abduction velocity.

photographed. Saccadic latency and velocity were measured off-line from the photographic record with a digitising pad and microcomputer. The subject sat in front of a visual display unit on which a BBC microcomputer produced a randomly moving white spot which appeared in the centre of the screen for a period of 2 to 4 seconds before moving horizontally $20^{\circ}$ to the right or to the left of the starting position. An average was taken of the latency and velocity of five saccades in each direction.

Definition of the normal range of saccadic latency and velocity Our normal values for saccadic latency and velocity have been published previously, ${ }^{11}$ derived from 85 subjects (44 males, 41 females) whose average age was 41 years (range 2068). Both latency and velocity differ for abduction and adduction and correlate with age. In this study the normal ranges for latency and velocity are defined by the $99 \%$ confidence intervals about the regressions upon age, as shown in the figure.

\section{(b) Evoked responses}

These were measured using standardised techniques. Visual evoked potentials were determined using a checkerboard reversal pattern stimulus, illumination 117 lux, full field $25^{\circ}$ individual checks 48 minutes. The normal range was defined by $99 \%$ confidence intervals about separate quadratic regression curves for males and females, weighted to take account of the variability of latency with age. ${ }^{12}$

The brainstem auditory evoked potential was measured using a monoaural click stimulus of $100 \mu$ s duration and alternate polarity (70 decibels above hearing level). Recording gain was $5 \mu \mathrm{V}$, low frequency filter $300 \mathrm{~Hz}$ and high frequency $3 \mathrm{kHz}$. The interwave latency of waves I, III and V and the ratio of the amplitudes of waves $I / V$ were the only parameters measured. ${ }^{13}$

\section{Statistical methods}

The chi-squared test with Yates' correction, and McNemar's test for matched data were used as appropriate. 


\section{Results}

\section{Saccadic eye movement recording}

Individual results for abduction and adduction, latency and velocity in relation to the $99 \%$ confidence intervals for healthy subjects are shown in the figure. The results in those patients showing one or more abnormality on electrophysiological testing are summarised in the table.

Abnormalities of saccadic movement were found in 21 patients of whom 14 had only one modality affected (seven latency, seven velocity); the remainder had abnormalities of both. Twelve of 14 abnormalities of saccadic latency could be explained by a disturbance of the gaze mechanism, with prolongation of abduction latency in one eye accompanied by prolongation of adduction latency in the other. Six patients had saccadic latency prolongation for gaze bilaterally, four for right gaze only and two for left gaze (one of whom, in addition, had an increased latency of adduction of the left eye).

Only three of the 14 patients with saccadic velocity abnormalities had gaze paresis alone, one to the right and two to the left. Two patients had reduced velocity of gaze (one to each side) together with reduction of adduction velocity in the ipsilateral eye (the "one and a half" syndrome.$^{14} \mathrm{An}$ isolated reduction of adduction velocity was present in two patients, and of abduction velocity in one (all on the left). An increased velocity of abduction was found in six patients, two in the right eye and four in the left eye. Increased abduction velocity was not associated with decreased contralateral adduction velocity, which would have been indicative of internuclear ophthalmoplegia. There was an association between reduced velocity and increased latency for the same movement (Chi-squared $=4 \cdot 36$, $\mathrm{p}<0.05$ ).

Although none of the patients had clinically definite abnormalities of eye movement, several had questionable nystagmus. However, suspicion of nystagmus was not confined to those with abnormal measurements of saccadic eye movements. Three patients were thought to have had internuclear ophthalmoplegia in the past, though there was no clinical evidence of this when they were examined in this study. All three had abnormalities of saccadic eye movement measurements, with reduction of adduction velocity in the direction of gaze which had previously been affected clinically; in addition one patient had a gaze palsy to the opposite side ("one and a half syndrome"), and another had

Table Results of SEMR (Velocity and latency), VEP, and BAER in the 30 patients in whom one or more test was abnormal

\begin{tabular}{|c|c|c|c|c|c|c|}
\hline \multirow[b]{2}{*}{ Sex } & \multirow[b]{2}{*}{ Age (yr) } & \multicolumn{4}{|c|}{$S E M R$} & \multirow[b]{2}{*}{$B A E R$} \\
\hline & & Clinical signs & Velocity & Latency & $V E P$ & \\
\hline $\begin{array}{l}\text { Female } \\
\text { Female } \\
\text { Female } \\
\text { Female } \\
\text { Male } \\
\text { Female } \\
\text { Female } \\
\text { Female } \\
\text { Male } \\
\text { Male } \\
\text { Female } \\
\text { Male } \\
\text { Male } \\
\text { Male } \\
\text { Male } \\
\text { Female } \\
\text { Male } \\
\text { Female } \\
\text { Male } \\
\text { Male } \\
\text { Female } \\
\text { Male } \\
\text { Female } \\
\text { Female } \\
\text { Female } \\
\text { Male } \\
\text { Male } \\
\text { Male } \\
\text { Female } \\
\text { Female } \\
\text { Totals (\%) }\end{array}$ & $\begin{array}{l}22 \\
26 \\
30 \\
32 \\
34 \\
56 \\
38 \\
49 \\
50 \\
51 \\
52 \\
53 \\
54 \\
55 \\
55 \\
56 \\
56 \\
57 \\
58 \\
58 \\
59 \\
59 \\
61 \\
63 \\
64 \\
67 \\
70 \\
70 \\
73 \\
82\end{array}$ & $\begin{array}{l}\text { L nyst } \\
\text { ? INO } \\
\text { R INO } \\
\text { B nyst } \\
\text { B nyst } \\
\text { L INO } \\
\text { L nyst } \\
\text { B nyst } \\
\text { L nyst }\end{array}$ & $\begin{array}{l}+ \\
+ \\
+ \\
+ \\
+ \\
+ \\
+ \\
+ \\
+ \\
+ \\
+ \\
+ \\
+ \\
+\end{array}$ & $\begin{array}{l} \\
+ \\
+ \\
+ \\
+ \\
+ \\
+ \\
+ \\
+ \\
+ \\
+ \\
+ \\
+ \\
+ \\
+ \\
+ \\
+\end{array}$ & $\begin{array}{l}+ \\
+ \\
+ \\
+ \\
+ \\
+ \\
+ \\
17(32) \text { 21(40) }\end{array}$ & $\begin{array}{l}+ \\
+ \\
+ \\
+\end{array}$ \\
\hline
\end{tabular}

$(B=$ bilateral, $L=$ left, $R=$ right, nyst $=$ nystagmus, $I N O=$ inter-nuclear ophthalmoplegia, $+=$ a positive test result). 
reduced abduction velocity to the same side giving a gaze paresis to that side.

\section{Visual evoked potentials}

Seventeen patients had prolongation of P100 latency, 14 as a unilateral finding, eight on the right and six on the left, and three bilaterally. Prolongation of VEP latency was significantly associated with increased latency of saccadic eye movement (Chi-squared = $4 \cdot 04, p<0.05)$.

\section{Brainstem auditory evoked responses}

Nine patients had abnormalities of the BAER. These were a heterogeneous group: two patients had no wave $\mathrm{V}$, one on the left and the other bilaterally; another two patients had no wave III unilaterally, one on each side. A single patient had both waves III and V missing on the left. Of the remaining patients, in one case the I/V ratio was increased on the left, two other patients had abnormal III-V latencies on the right but in one it was associated with an absent wave $V$ on the left. The final patient had left-sided increases of the III-V latency as well as increase in the $\mathrm{I} / \mathrm{V}$ ratio. No association was found between abnormal saccadic eye movement recordings and abnormalities of the BAER.

\section{Comparative sensitivity of different tests}

Thirty out of a total of $53(57 \%)$ patients with noncompressive myelopathy had one or more abnormal test result (table), thus demonstrating dissemination of pathology and supporting a diagnosis of "clinically probable multiple sclerosis". ${ }^{\text {is }}$ McNemar's test was used to compare sensitivities: SEMR was more sensitive than BAER $(40 \% \vee 17 \%, p<0.01)$ but no significant differences in sensitivity were found between SEMR and VEP $(40 \%$ v $32 \%)$, or between VEP and BAER ( $32 \% \mathrm{v} 17 \%)$. If only two of the three tests were used, the number of patients with abnormal electrophysiological tests fell from 30 to 29,23 , or 21 for the omission of BAER, VEP or SEMR respectively.

\section{Discussion}

Saccadic eye movement recording (SEMR) was of considerable diagnostic value in our study group of patients with non-compressive myelopathy, demonstrating dissemination of pathology in $40 \%$. The detection rate increased from $40 \%$, using VEP and BAER only, to $57 \%$ in conjunction with SEMR, which was shown to be more sensitive than BAER. Indeed BAER could have been omitted with little effect on the results, being solely responsible for diagnosis in only one patient.

Knezevic et $\mathrm{al}^{7}$ found that the proportion of patients in whom a diagnosis of multiple sclerosis

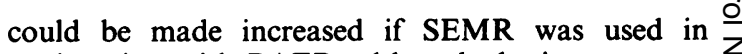
conjunction with BAER, although the increase was $\mathbb{D}$ only marked in the "probable" multiple sclerosis group. As in this study the sensitivity of SEMR was of greater than BAER, with 16 cases showing abnor- $\overline{\hat{\theta}}$ malities with SEMR alone compared with only three 0 with BAER alone. Sanders $e t a^{8}$ recommended the use of both SEMR and BAER to optimise detection of brainstem disease although SEMR detected the larger number of asymptomatic abnormalities.

The test itself is quick and easy to perform, well $\overrightarrow{\bar{N}}$ tolerated by patients, and has good reproducibility. ${ }^{1} \frac{7}{0}$ It may be improved by conversion of the analogue $\frac{\mathrm{C}}{\mathrm{O}}$ signal to digital form which allows on-line analysis by $\frac{\overline{\bar{D}}}{\bar{D}}$ computer ${ }^{1617}$ Such a system would make it possible to $\vec{\nabla}$ measure other eye movements, such as the velocity of smooth pursuit.

Our results are not exactly comparable with studies $\overrightarrow{0}$ of similar patients in which other types of eye $\overrightarrow{\vec{H}}$ movement, such as the velocity of smooth pursuit, $\vec{\omega}$ have been measured. ${ }^{56}$ Reulen et al $^{6}$ claimed that qualitative assessment of the accuracy of saccadic movement was also of value. We were unable to or confirm this as we found that abnormalities such as? hypometria occurred as frequently in the contreb. population as in our patients. Solingen et $a l^{4}$ also $\overrightarrow{\vec{O}}$ found a mild degree of fixation instability in norm雨 subjects.

The large control population (85) that we useg enabled us to recognise the importance in defining abnormal results of the patient's age (but not sex), ang ? the type of movement, whether adduction or abdur tion. A variety of abnormalities of saccadic ese movement was found. Nearly all latency abnort $\vec{D}_{0}$ malities produced a delay in reaction time of gaze to one or both sides rather than delaying individual 0 movements of either eye. The association between VEP abnormalities, which were unilateral in the majority of cases, and latency abnormalities, which

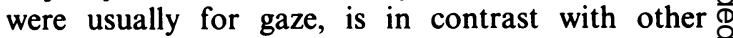
reports $^{618}$ and may indicate that lesions in the primary visual pathway influence saccadic latency.

Velocity abnormalities were heterogeneous. Slowing of adduction (medial longitudinal fasiculus syndrome) was common, but when it was present we did not find that it was usually bilateral, in contrast with clinical observation ${ }^{2}$ and other oculographic studies. ${ }^{19}$ More frequently it was part of a gaze paresis, 3 and when found bilaterally was associated with a contralateral gaze paresis as in the "one and a half" syndrome. ${ }^{14}$ This syndrome has only rarely been

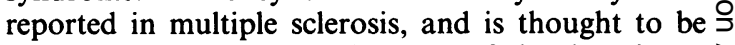
caused by unilateral involvement of the dorsal teg- $\rightarrow$ mentum of the lower pons. ${ }^{20}$ However, abnormalities of the velocity of either adduction or abduction may be $N$ found in internuclear ophthalmoplegia, ${ }^{2122}$ pre- 
sumably depending on the balance of excitation and inhibition of the medial recti. Disease of the medial longitudinal fasciculus may therefore explain the abnormalities of saccadic velocity observed in this study and it is unnecessary to invoke lesions in other sites.

A most unusual finding was an isolated increase in abduction velocity in six cases. This abnormality is unexplained; it is unlikely to result from disease of the medial longitudinal fasciculus since it was not associated with slowed adduction in the same or the opposite eye. As far as we are aware, increased velocity of abduction has not been reported in multiple sclerosis, but has been described in myaesthenia gravis. ${ }^{23}$

In conclusion, saccadic eye movement recording is a sensitive test for the "second lesion" in multiple sclerosis, reflecting the high incidence of oculomotor abnormalities in this disease. The technique readily enables analysis of clinical and subclinical disorders of eye movement.

\section{References}

1 Matthews WB. Symptoms and signs (contd). In: Matthews WB, Acheson ED, Batchelor JR, Weller RO, eds. McAlpine's Multiple Sclerosis. New York: Churchill Livingstone 1985: $119-45$.

2 Cogan, DG. Internuclear ophthalmoplegia, typical and atypical. Arch Ophthalmol 1970;84:583-9.

3 Young LR, Sheena D. Methods and designs. Survey of eye movement recording methods. Behav Res Methods Instrumentation. 1975;7:397-429.

4 Solingen LD, Baloh RW, Myers L, Ellison G. Subclinical eye movement disorders in patients with multiple sclerosis. Neurology 1977;27:614-9.

5 Mastaglia FL, Black JL, Collins DWK. Quantitative studies of saccadic and pursuit eye movements in multiple sclerosis. Brain 1979;102:817-34.

6 Reulen JPH, Sanders EACM, Hogenhuis LAH. Eye movement disorders in multiple sclerosis and optic neuritis. Brain 1983; 106: $121-40$.

7 Knezevic W, Mastaglia JL, Black JL, Collins DWK. Brainstem
Auditory evoked responses and quantitative saccade studies in multiple sclerosis: a comparative evaluation. Clin Exp Neurol 1984;20:175-9.

8 Sanders EACM, Reulen JPH, Hogenhuis LAG, van der Velde EA. Brainstem involvement in multiple sclerosis: a clinical and electrophysiological study. Acta Neurol Scand 1985;71:54-61.

9 Hubbe P, Dam AM. Spastic Paraplegia of unknown origin. Acta Neurol Scand 1973;49:536-42.

10 Paty DW, Blume WT, Brown WF, Jaatoul N, Kertesz A, McInnes W. Chronic progressive myelopathy, investigation with CSF electrophoresis, evoked potentials and CT scan. Ann Neurol 1979;6:419-24.

11 Pitt MC, Rawles JM. The effect of ageing on saccadic latency and velocity. Neuro-ophthalmology 1988;8:123-9.

12 Pitt MC, Daldry SJ. The use of weighted quadratic regression for the study of latencies of the P100 component of the visual evoked potential. Electroencephalogr Clin Neurophysiol 1988; 71:150-2.

13 Chiappa KH, Harrison JL, Brooks KB, Young RR. Brainstem auditory evoked responses in 200 patients with multiple sclerosis. Ann Neurol 1980;7:135-43.

14 Fisher CM. Some neuro-ophthalmological observations. J Neurol Neurosurg Psychiatry. 1967;30:383-92.

15 Poser CM, Paty DW, Scheinberg L, et al. New diagnostic criteria for MS: guidelines for research protocols. Ann Neurol 1983; 13:227-31.

16 Balch RW, Langhofer L, Honrubia V, Yee RD. On-line analysis of eye movements using a digital computer. Aviat Space Environ Med 1980;51:563-7.

17 Smith AT, Bittencourt PRM, Lloyd DSL, Richens A. An efficient technique for determining characteristics of saccadic eye movements using a microcomputer. J Biomed Eng 1981;3: 39-43.

18 Ochs AL, Hoyt WF, Stark L, Patchman MA. Saccadic initiation time in multiple sclerosis. Ann Neurol 1978;4:578-9.

19 Feldon SE, Hoyt WF, Stark L. Disordered inhibition in internuclear ophthalmoplegia. Analysis of eye movement recordings with computer simulations. Brain 1980;103:113-37.

20 Pierrot-Deseilligny CH, Chain F, Serdau M, Gray F, Lhermitte F. The 'one-and-a-half syndrome: electro-oculographic analyses of five cases with deductions about the physiological mechanisms of lateral gaze. Brain 1981;104:665-99.

21 Bird AC, Leech J. Internuclear ophthalmoplegia. An electrooculographic study of peak angular saccadic velocities. $\mathrm{Br} J$ Ophthalmol 1976;60:645-50.

22 Kirkham TH, Katsarkas A. An electro-oculographic study of internuclear ophthalmoplegia. Ann Neurol 1977;2:385-92.

23 Oohira A, Goto K, Sato Y, Ozawa T. Saccades of supernormal velocity. Adaptive response to ophthalmoplegia in a patient with myasthenia gravis. Neuro-ophthalmology 1987;7:203-9. 